\title{
DISSONANT POINTS AND THE REGION OF INFLUENCE OF NON-SADDLE SETS
}

\author{
HÉCTOR BARGE AND JOSÉ M.R. SANJURJO
}

\begin{abstract}
The aim of this paper is to study dynamical and topological properties of a flow in the region of influence of an isolated non-saddle set. We see, in particular, that some topological conditions are sufficient to guarantee that these sets are attractors or repellers. We study in detail the existence of dissonant points of the flow, which play a key role in the description of the region of influence of a non-saddle set. These points are responsible for much of the dynamical and topological complexity of the system. We also study non-saddle sets from the point of view of the Conley index theory and consider, among other things, the case of flows on manifolds with trivial first cohomology group. For flows on these manifolds, dynamical robustness is equivalent to topological robustness. We carry out a particular study of 2-dimensional flows and give a topological condition which detects the existence of dissonant points for flows on surfaces. We also prove that isolated invariant continua of planar flows with global region of influence are necessarily attractors or repellers.
\end{abstract}

2010 MSC: 37C75, 37B30.

Non-saddle set, region of influence, dissonant point, Conley index, homoclinic orbit, robustness.

\section{INTRODUCTION}

This paper is devoted to the study of a flow near a compact invariant set. This was one of the first classical subjects dealt with by H. Poincaré, I. Bendixson, A. Andronov and S. Lefschetz at the beginning of the qualitative theory of differential equations, with further contributions by authors such as D.M. Grobman, P. Hartman, J.K. Hale and A. Stokes and many others. T. Ura and I. Kimura were able to provide in [42] a rather general description. Wazewski's and Conley's index theories can be considered an important chapter of this general topic since they deal essentially with local properties of the flow near (isolated) invariant sets. The local character of the flow is, to a large extent, determined by the perturbation properties of attraction and repulsion induced by the invariant set in its vicinity and, for this reason, we study in detail its region of influence. Isolated non-saddle sets are important in this context since they have an open region of influence. Moreover, this region has a particularly nice structure near the compactum since it is organized into a purely attracted part and a purely repelled part, where the properties of attraction and repulsion are uniform. In other words, isolated non-saddle sets have isolating blocks consisting of the asymptotic (positive and negative) parts only. Saddle and non-saddle sets were first studied by N.P. Bhatia [4] and by T. Ura [41] but, according to Ura, they were introduced before by P. Seibert in an oral communication. The theory of non-saddle sets can be considered as a general theory of

THE AUTHORS ARE SUPPORTED BY MINECO (MTM2012-30719). THE FIRST AUTHOR IS ALSO SUPPORTED BY THE FPI GRANT BES-2013-062675. 
stability and attraction, which extends the classical one and encompasses recent developments such as the theory of unstable attractors with no external explosions [1, 25, 33.

We study in the paper the topological aspects of non-saddle sets and of their region of influence. We see, in particular, that some topological conditions are sufficient to guarantee that these sets are attractors or repellers. We examine the general structure of a flow having an isolated non-saddle set. This structure can be considerably more complicated than the one for attractors and repellers and even than the one for unstable attractors with no external explosions. We show that the reason for this complexity lies in the existence of dissonant points in the region of influence of the non-saddle set. We illustrate this phenomenon with an example based on a saddle-node bifurcation in the torus. We identify topological conditions which prevent the existence of dissonant points. For instance, flows on connected closed manifolds $M$ with trivial first cohomology group $H^{1}(M)$ do not have dissonant points and the region of influence of the non-saddle set has a nice description. We also give some conditions, in terms of the Conley index of the invariant set, for it to be non-saddle. This result is a source of many interesting examples of flows on manifolds admitting all degrees of dynamical complexity. We carry out a particular study of 2-dimensional flows. We describe the structure of a class of non-saddle sets in the torus. We find a condition, involving the Euler characteristic of the region of influence, to detect the existence of dissonant points of flows on surfaces. We prove that isolated invariant continua of planar flows with global region of influence are attractors or repellers (in particular, they enjoy either positive or negative stability). Some results about existence of fixed points for planar flows are also established. Finally, we study the continuation properties of non-saddle sets for parametrized families of flows. We prove that, for flows on compact connected and oriented differentiable manifolds with trivial 1-dimensional cohomology, dynamical robustness is equivalent to topological robustness and, as a consequence, the preservation of non-saddleness can be detected by topological means. It can be seen that non-saddle sets are involved in generalized Andronov-Poincaré-Hopf bifurcations (in the sense of [37]) but this will be the subject of a different paper. Our point of view is mainly topological and has deep connections with the Conley index theory. We stress the richness of the topological aspects of dynamical systems and differential equations, often presenting unexpected and strange properties. As was remarked by Kennedy and Yorke in [20] "bizarre topology is natural in dynamical systems."

Through the paper we shall consider flows $\varphi: M \times \mathbb{R} \rightarrow M$ defined on locally compact metric spaces. However, we will often focus on the particular cases of flows defined on locally compact ANRs and $n$-dimensional manifolds. A metrizable space is said to be an absolute neighborhood retract (or $M \in A N R$ ) if for every homeomorphism $h$ mapping $M$ onto a closed subset $h(M)$ of a metrizable space $X$ there is a neighborhood $U$ of $h(M)$ in $X$ such that $h(M)$ is a retract of $U$. The class of ANRs includes a lot of nice spaces such as polyhedra, CW-complexes, manifolds, etc... Besides, open subsets of ANRs and retracts of ANRs are also ANRs. For more information about the theory of retracts see [17].

A form of homotopy theory, namely shape theory, which is the most suitable for the study of global topological properties in dynamics, will be occasionally used. Although a deep knowledge of shape theory is not necessary to understand the paper we recommend to the reader Borsuk's monography [7] for an exhaustive treatment of the subject and [19, 29, 31, 34, 35, 36, 38, 43] for a concise introduction and some applications to dynamical systems. 
We make use of some notions of algebraic topology. A good reference for this material is the book of Spanier [39]. We will use the notation $H_{*}$ and $H^{*}$ for the singular homology and cohomology respectively and we will denote by $\check{H}^{*}$ the Čech cohomology, all of them with integer coefficients unless otherwise specified. We recall that Čech and singular cohomology theories agree on ANRs and, from this fact, combined with a simple argument involving the long exact sequences of Cech and singular cohomology of a pair, the natural homomorphism between both cohomologies and the five lemma, it can be proved that it also holds for pairs of ANRs. Another nice property of Cech cohomology is that it is a shape invariant. A recent paper showing some applications of homological techniques to dynamical systems is [21.

If a pair of spaces $(X, A)$ satisfies that its cohomology $\check{H}^{k}(X, A)$ is finitely generated for each $k$ and is non-zero only for a finite number of values of $k$, its Euler characteristic is defined as

$$
\chi(X, A)=\sum_{k \geq 0}(-1)^{k} \operatorname{rk} \check{H}^{k}(X, A) .
$$

A useful property of the Euler characteristic which can be found in [39] is that

$$
\chi(X)=\chi(X, A)+\chi(A) .
$$

A different Euler characteristic can be defined by using singular homology and cohomology (both produce the same result by the Universal Coefficients Theorem) in the same way. This singular Euler characteristic agrees with the Euler characteristic previously defined for ANRs and pairs of ANRs by the previous remarks.

The main reference for the elementary concepts of dynamical systems will be [5] but we also recommend [30, 26, 27]. In the sequel we shall use the notation $\gamma(x)$ for the trajectory of the point $x$, i.e. $\gamma(x)=\{x t \mid t \in \mathbb{R}\}$. Similarly for the positive semi-trajectory $\gamma^{+}(x)=\{x t \mid$ $\left.t \in \mathbb{R}^{+}\right\}$and the negative-semitrajectory $\gamma^{-}(x)=\left\{x t \mid t \in \mathbb{R}^{-}\right\}$. By the omega-limit of a point $x$ we understand the set $\omega(x)=\bigcap_{t>0} \overline{x[t, \infty)}$ while the negative omega-limit is the set $\omega^{*}(x)=\bigcap_{t<0} \overline{x(-\infty, t]}$. On the other hand, the positive prolongational limit set of a point $x$ is the set

$$
J^{+}(x)=\bigcap_{U \in \mathcal{E}(x), t>0} \overline{U[t, \infty)}
$$

where $\mathcal{E}(x)$ denotes the system of neighborhoods of the point $x$. The negative prolongational limit set of a point $x, J^{-}(x)$ is defined in an analogous fashion. Besides, we will need to introduce the concept of two-sided prolongational limit set

Definition 1. Given $x \in M$, the two-sided prolongational limit set of $x$, is defined to be

$$
\begin{array}{r}
J^{*}(x):=\left\{(y, z) \in M \times M \mid \text { there exist } x_{n} \rightarrow x, t_{n} \rightarrow \infty, s_{n} \rightarrow-\infty\right. \\
\text { such that, } \left.x_{n} t_{n} \rightarrow y \text { and } x_{n} s_{n} \rightarrow z\right\} .
\end{array}
$$

It is easy to see that $J^{*}$ is closed in $M \times M$ and that $(y t, z s) \in J^{*}(x)$ for every $(y, z) \in J^{*}(x)$ and every $t, s \in \mathbb{R}$.

Another notion which will be useful is that of parallelizable flow. A flow $\varphi: M \times \mathbb{R} \rightarrow M$ is said to be parallelizable if there exists a subset $S \subset M$ such that the map $h: S \times \mathbb{R} \rightarrow M$ defined by $(x, t) \mapsto x t$ is a homeomorphism. The set $S$ is called a section of $\varphi$ and a direct consequence of the definition is that it is a strong deformation retract of $M$. 
An important class of invariant compacta is the so-called isolated invariant sets (see [8, 9, 12 for details). These are compact invariant sets $K$ which possess an isolating neighborhood, i.e. a compact neighborhood $N$ such that $K$ is the maximal invariant set in $N$.

A special kind of isolating neighborhoods will be useful in the sequel, the so-called isolating blocks, which have good topological properties. More precisely, an isolating block $N$ is an isolating neighborhood such that there are compact sets $N^{i}, N^{o} \subset \partial N$, called the entrance and the exit sets, satisfying

(1) $\partial N=N^{i} \cup N^{o}$;

(2) for each $x \in N^{i}$ there exists $\varepsilon>0$ such that $x[-\varepsilon, 0) \subset M-N$ and for each $x \in N^{o}$ there exists $\delta>0$ such that $x(0, \delta] \subset M-N$;

(3) for each $x \in \partial N-N^{i}$ there exists $\varepsilon>0$ such that $x[-\varepsilon, 0) \subset \stackrel{\circ}{N}$ and for every $x \in \partial N-N^{o}$ there exists $\delta>0$ such that $x(0, \delta] \subset \stackrel{\circ}{ }$.

These blocks form a neighborhood basis of $K$ in $M$. If the flow is differentiable, the isolating blocks can be chosen to be differentiable manifolds which contain $N^{i}$ and $N^{o}$ as submanifolds of their boundaries and such that $\partial N^{i}=\partial N^{o}=N^{i} \cap N^{o}$. In particular, for flows defined on $\mathbb{R}^{2}$, the exit set $N^{o}$ is a disjoint union of a finite number of intervals $J_{1}, \ldots, J_{m}$ and circumpherences $C_{1}, \ldots, C_{n}$ and the same is true for the entrance set $N^{i}$.

Given an isolating block $N$ of an isolated invariant set $K$, its positively invariant part is the set $N^{+}=\left\{x \in N \mid \gamma^{+}(x) \subset N\right\}$. In an analogous way, the negatively invariant part of $N$, is the set $N^{-}=\left\{x \in N \mid \gamma^{-}(x) \subset N\right\}$. It is clear that $N^{+}$and $N^{-}$are, respectively, positively and negatively invariant closed subsets of $N$ (and hence compact) whose intersection is $K$.

Let $K$ be an isolated invariant set, its Conley index $h^{+}(K)$ is defined as the pointed homotopy type of the topological space $\left(N / N^{o},\left[N^{o}\right]\right)$. It is also possible to define its negative Conley index $h^{-}(K)$, which agrees with the Conley index of $K$ for the reverse flow, as the pointed homotopy type of the topological space $\left(N / N^{i},\left[N^{i}\right]\right)$. A weak version of the Conley index which will be useful for us is the cohomological index defined as $C H_{+}^{*}(K)=$ $\check{H}^{*}\left(h^{+}(K)\right)$. The negative cohomological index $C H_{-}^{*}(K)$ is defined in an analogous fashion. Observe that, by the strong excision property of Čech cohomology, $C H_{+}^{*}(K) \cong \check{H}^{*}\left(N, N^{o}\right)$ and $C H_{-}^{*}(K) \cong \check{H}^{*}\left(N, N^{i}\right)$. Our main references for the Conley index theory are [8, 10, 32]. We also recommend the survey [18] where some connections with classical Morse theory and Brouwer degree are stated.

We will deal through the paper with a special class of invariant sets, the so-called nonsaddle sets [4]. A compact invariant set $K$ is said to be saddle if it admits a neighborhood $U$ such that every neighborhood $V$ of $K$ contains a point $x \in V$ with $\gamma^{+}(x) \nsubseteq U$ and $\gamma^{-}(x) \nsubseteq U$. Otherwise we say that $K$ is non-saddle. For instance, attractors (asymptotically stable sets), repellers (negatively asymptotically stable sets) and unstable attractors with no external explosions (see [1, 25, 33]) are non-saddle sets.

If $K$ is an invariant set, its stable manifold $\mathcal{A}(K)$ is the set of all points $x \in M$ such that $\emptyset \neq \omega(x) \subset K$. Similarly, the unstable manifold $\mathcal{R}(K)$ is the set of all points $x \in M$ such that $\emptyset \neq \omega^{*}(x) \subset K$. The region of influence of an invariant set $K$ is the set $\mathcal{I}(K)=\mathcal{A}(K) \cup \mathcal{R}(K)$.

An isolated non-saddle set is said to be simple if $\mathcal{A}(K) \cap \mathcal{R}(K)=K$. It is well known that the inclusion $i: K \hookrightarrow \mathcal{A}(K)$ of an attractor in its basin of attraction is a shape equivalence, and the same is true for the inclusion $j: K^{\prime} \hookrightarrow \mathcal{R}\left(K^{\prime}\right)$ of a repeller in its basin of repulsion 
[19, 38. By combining these two facts it is easy to see that if $K$ is simple, the inclusion $i: K \hookrightarrow \mathcal{I}(K)$ is a shape equivalence.

We will also make use of a classical result of C. Gutiérrez about smoothing of 2-dimensional flows.

Theorem 2 (Gutiérrez [15]). Let $\varphi: M \times \mathbb{R} \rightarrow M$ be a continuous flow on a compact $C^{\infty}$ 2-manifold $M$. Then there exists a $C^{1}$ flow $\psi$ on $M$ which is topologically equivalent to $\varphi$. Furthermore, the following conditions are equivalent:

(1) any minimal set of $\varphi$ is trivial;

(2) $\varphi$ is topologically equivalent to a $C^{2}$ flow;

(3) $\varphi$ is topologically equivalent to a $C^{\infty}$ flow.

By a trivial minimal set we understand a fixed point, a closed trajectory or the whole manifold if $M$ is the 2-dimensional torus and $\varphi$ is topologically equivalent to an irrational flow. We readily deduce from Gutiérrez' Theorem applied to the Alexandrov compactification of the plane that continuous flows $\varphi: \mathbb{R}^{2} \times \mathbb{R} \rightarrow \mathbb{R}^{2}$ are topologically equivalent to $C^{\infty}$ flows.

We use some basic results about planar vector fields through the paper. Two good references covering this material are the book of Hirsch, Smale and Devaney [16] and the monograph of Palis and de Melo [26].

Notice that to avoid trivial cases when we consider an (isolated) invariant set $K$ it will be implicit that $\emptyset \neq K \subsetneq M$ unless otherwise specified.

The authors are grateful to J.M. Montesinos-Amilibia and J.J. Sánchez-Gabites for useful and inspiring conversations.

\section{TOPOLOGICAL ASPECTS OF NON-SADDLE SETS}

In this section we will study some topological aspects of a flow $\varphi: M \times \mathbb{R} \rightarrow M$ defined on a locally compact metric space having an isolated non-saddle set. For instance, we will give a characterization of non-saddleness in terms of influence-like properties and we will also characterize the shape of those non-separating isolating non-saddle sets which are neither attractors nor repellers in the torus.

We start by stating a well-known result about isolating blocks of isolated non-saddle sets whose proof we include here for the sake of completeness.

Proposition 3. Every isolated non-saddle set admits a basis of isolating blocks of the form $N=N^{+} \cup N^{-}$.

Proof. Since any isolated invariant compactum admits a neighborhood basis comprised of isolating blocks, it would be sufficient to prove that given an arbitrary isolating block $N$ of an isolated non-saddle set $K$, it contains an isolating block $N_{0}=N_{0}^{+} \cup N_{0}^{-}$.

Let $N$ be an isolating block of the isolated non-saddle set $K$. Then, $K$ being non-saddle, there exists a neighborhood $V \subset N$ of $K$ such that for each $x \in V$, either $\gamma^{+}(x) \subset N$ or $\gamma^{-}(x) \subset N$. As a consequence, the compact subset $N_{0}=N^{+} \cup N^{-}$is an isolating neighborhood of $K$ since $V \subset N_{0}$ and it is contained in the isolating block $N$. Moreover, $N_{0}$ is an isolating block. To see this we prove that $\partial N_{0} \subset \partial N$. Suppose that there exists $x \in \partial N_{0}-\partial N$. Then, $x \in N^{\circ}$ and there exists a sequence $x_{n}$ in $N-\left(N^{+} \cup N^{-}\right)$such that $x_{n} \rightarrow x$. Suppose that $x \in N^{+}$, otherwise the argument is analogous. Let $t_{n}$ be a sequence 
of positive times,$t_{n} \rightarrow \infty$. Then, $x t_{n} \rightarrow K$ as $n \rightarrow \infty$ and, maybe after passing to a subsequence, $x_{n} t_{n} \rightarrow K$ as $n \rightarrow \infty$. Besides, the choice of $x_{n}$ ensures that neither $\gamma^{+}\left(x_{n} t_{n}\right)$ nor $\gamma^{-}\left(x_{n} t_{n}\right)$ are contained in $N$ leading to a contradiction with $K$ being non-saddle. Indeed, $x_{n} t_{n}$ cannot be in $N^{-}$since $x_{n}$ leaves $N$ in negative time, being $x_{n} \in N-\left(N^{+} \cup N^{-}\right)$and, since $t_{n}>0$ so does $x_{n} t_{n}$. We see that $x_{n} t_{n}$ cannot be in $N^{+}$. Suppose, to get a contradiction, that $x_{n} t_{n} \in N^{+}$for almost all $n$. Then, there exists a sequence $0<s_{n}<t_{n}$ such that $x_{n} s_{n} \in N^{o}$ for all $n$. Moreover, the sequence $s_{n}$ must be bounded. If not, $x_{n} s_{n}$ would have a subsequence convergent to a point $z \in N^{-}$and, as a consequence, $x_{n}$ would have a subsequence such that the positive semi-trajectory of each one of its elements gets arbitrarily close to $K$ before leaving $N$ in contradiction with its non-saddleness. Thus, we may assume that $s_{n} \rightarrow s_{0} \geq 0$ and, hence, $x_{n} s_{n} \rightarrow x s_{0}$. Since $x_{n} s_{n} \in N^{o}$ for each $n$, so does $x s_{0}$. However, $x s_{0} \in N^{+}$ which has empty intersection with $N^{o}$ and we get a contradiction. Therefore, $\partial N_{0}$ agrees with $\partial N \cap N_{0}$ and the compact subsets $N_{0}^{i}=N^{+} \cap \partial N$ and $N_{0}^{o}=N^{-} \cap \partial N$ are respectively an entrance and an exit set ensuring that $N_{0}$ is an isolating block.

Remark 4. It is not difficult to see that the isolating block $N_{0}$ defined in the proof of Proposition 3 agrees with the union of those components of $N$ which contain some component of $K$. It follows from this fact that every connected isolating block of a connected non-saddle set is of the form $N^{+} \cup N^{-}$.

A nice consequence of Proposition 3 is that for flows defined on locally compact ANRs, isolated non-saddle sets have the shape of finite polyhedra and hence, finitely generated Čech homology and cohomology. Moreover, it can be seen that if $N=N^{+} \cup N^{-}$, the inclusion $i: K \hookrightarrow N$ is a shape equivalence. These results were obtained in [13].

We see that, in analogy with the basin of attraction of an attracting set (attractors and unstable attractors), the region of influence of an isolated non-saddle set is an open set.

Proposition 5. If $K$ is an isolated non-saddle compactum then $\mathcal{I}(K)$ is an open neighborhood of $K$.

Proof. Given an isolating neighborhood of $K$ of the form $N=N^{+} \cup N^{-}$, it must be contained in $\mathcal{I}(K)$, hence $\mathcal{I}(K)$ is a neighborhood of $K$. On the other hand, if $x \in \mathcal{I}(K)$ and, say, $\emptyset \neq \omega(x) \subset K$, then there is a neighborhood $U$ of $x$ and a $t_{0} \geq 0$ such that $U t_{0}$ is contained in $N^{+}$. Hence for every $y \in U t_{0}$ we have that $\emptyset \neq \omega(y) \subset K$ and, as a consequence, the same thing happens for every $z \in U$. Therefore $\mathcal{I}(K)$ is open.

However, in contrast with the case of attracting sets, the converse does not necessarily hold.

Remark 6. There are isolated saddle continua $K$ such that $\mathcal{I}(K)$ is an open neighborhood of $K$, hence this property does not characterize non-saddleness. For instance, consider the Mendelson's flow on the plane [24], see Figure 1.

Then $K=\left\{p_{2}\right\}$ is a saddle set (in fact, an unstable attractor) and its region of influence (region of attraction in this case) is $\mathbb{R}^{2}-\left\{p_{1}\right\}$.

The next result gives a sufficient condition for a non-saddle set to be either an attractor or a repeller. 


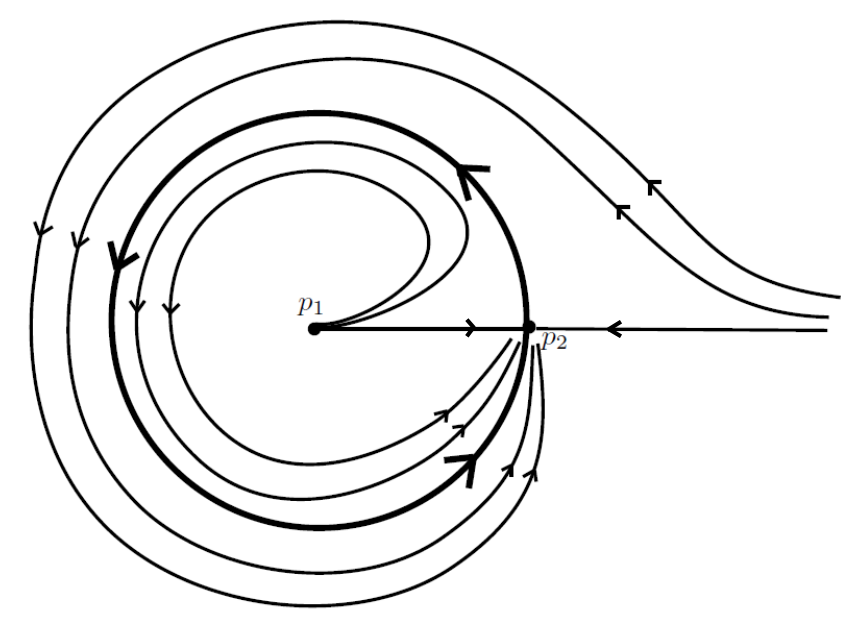

Figure 1. Mendelson's attractor

Proposition 7. Let $K$ be an isolated non-saddle continuum of a flow on a locally compact metric space $M$. Suppose that $K$ has arbitrarily small neighborhoods in $M$ which are not disconnected by $K$. Then $K$ is either an attractor or a repeller. In particular, if $M$ is an orientable $n$-dimensional manifold with $n>1$ and $K$ has trivial shape or, more generally, if $\check{H}^{n-1}(K)=\{0\}$, then $K$ is either an attractor or a repeller.

Proof. Consider a connected isolating block $N$ of $K$ of the form $N=N^{+} \cup N^{-}$, which is contained in $\mathcal{I}(K)$, and let $W$ be a neighborhood of $K$ such that $W \subset N$ and $W$ is not disconnected by $K$. Then either $W \cap\left(N^{+}-K\right)$ or $W \cap\left(N^{-}-K\right)$ is empty. In the first case $K$ is a repeller and in the second case an attractor. If $M$ is an orientable $n$-manifold then, by Alexander duality, $H_{1}(M, M-K) \cong \check{H}^{n-1}(K)$ and, by excision, $H_{1}(\stackrel{\circ}{N}, \stackrel{\circ}{N}-K) \cong$ $H_{1}(M, M-K)$. Thus, if $K$ has trivial shape or more generally $\check{H}^{n-1}(K)=\{0\}$ from the terminal part of the exact homology sequence of the pair $(\stackrel{\circ}{N}, \stackrel{\circ}{N}-K)$

$$
\ldots \rightarrow H_{1}(\stackrel{\circ}{N}, \stackrel{\circ}{N}-K)=\{0\} \rightarrow \widetilde{H}_{0}(\stackrel{\circ}{N}-K) \rightarrow \widetilde{H}_{0}(\stackrel{\circ}{N})=\{0\}
$$

we get that $\widetilde{H}_{0}(\stackrel{\circ}{N}-K)=\{0\}$. As a consequence $\stackrel{\circ}{N}$ is not disconnected by $K$. Since $N$ can be taken arbitrarily small, this is a particular case of the situation considered before.

Throughout this paper we will often use the torus as a relevant phase space for flows which illustrate the main notions we introduce. The previous proposition, together with some classical results in Algebraic Topology, can be used to describe the topological structure of an important class of non-saddle sets in the torus.

Theorem 8. Suppose that $K$ is an isolated non-saddle continuum of a flow on the torus, $T$, such that $K$ does not separate $T$ and $K$ is neither an attractor nor a repeller. Then $K$ has the shape of a circle. Moreover, if $K$ does not contain fixed points it is either a limit cycle or homeomorphic to a closed annulus bounded by two limit cycles. 
Proof. We take coefficients in $\mathbb{Z}_{2}$. Since $K$ does not separate $T$ we have that $\widetilde{H}_{0}(T-K)=\{0\}$. By using the exact homology sequence of the pair $(T, T-K)$

$$
\ldots \rightarrow H_{1}(T) \rightarrow H_{1}(T, T-K) \rightarrow \widetilde{H}_{0}(T-K)=\{0\} \rightarrow \ldots
$$

and Alexander duality we get that $\operatorname{rk} \check{H}^{1}(K)=\operatorname{rk} H_{1}(T, T-K) \leq 2$. We will prove, arguing by contradiction, that, in fact, $\operatorname{rk} \check{H}^{1}(K) \neq 2$. Suppose $\operatorname{rk} \check{H}^{1}(K)=2$ and let $N=N^{+} \cup N^{-}$ be an isolating block of $K$. Consider the terminal part of the reduced homology long exact sequence of the pair $(T, N)$

$$
\begin{array}{r}
\{0\}=H_{2}(N) \rightarrow H_{2}(T)=\mathbb{Z}_{2} \rightarrow H_{2}(T, N) \rightarrow H_{1}(N) \rightarrow H_{1}(T) \rightarrow \\
H_{1}(T, N) \rightarrow \widetilde{H}_{0}(N)=\{0\}
\end{array}
$$

The homomorphism $H_{2}(T) \rightarrow H_{2}(T, N)$ is an isomorphism since $H_{2}(T, N)=H^{2}(T, N)$ by the Universal Coefficients Theorem and by Alexander duality $H^{2}(T, N)=\check{H}^{2}(T, K) \cong H_{0}(T-$ $K)$, which is $\mathbb{Z}_{2}$ since $K$ does not separate $T$. As a consequence, the homomorphism $H_{1}(N) \rightarrow$ $H_{1}(T)$ is injective and hence an isomorphism. Therefore, $H_{1}(T, N)=\{0\}$ and by excising $K, H_{1}(T-K, N-K)=\{0\}$. Then, from the homology long exact sequence of the pair $(T-K, N-K)$ we get $H_{0}(N-K) \cong H_{0}(T-K)=\mathbb{Z}_{2}$, i.e. $K$ does not separate $N$. Besides, $N$ can be chosen arbitrarily small, $N$ being an isolating block, and hence Proposition 7 ensures that it has to be an attractor or a repeller in contradiction with the assumption. As a consequence, rk $\check{H}^{1}(K)$ is either 0 or 1 and by [6, 11, 22, 23] $K$ has either trivial shape or the shape of a circle respectively. The first case is excluded by Proposition 7 and hence $K$ has the shape of a circle.

For the last part of the statement observe that $K$, having the shape of a circle, admits an isolating block $N=N^{+} \cup N^{-}$which is an annulus by [15] and [9]. The annulus $N$ can be embedded into $\mathbb{R}^{2}$ endowed with a smooth flow which preserves the dynamics in a small neighborhood of $K$ contained in $\stackrel{\circ}{N}$. This can be achieved by a slight modification of [33, Example 21] combined with Gutiérrez Theorem. Then, if $K$ does not have fixed points, by [2] the result follows.

Remark 6] shows that unstable attractors are not necessarily non-saddle. As a matter of fact we have the following characterization, whose proof is given in [38].

Proposition 9. Let $K$ be an unstable attractor of a flow. Then $K$ has no external explosions if and only if it is non-saddle.

In order to characterize non-saddle sets by influence-like properties we introduce the following notion first.

Definition 10. A point $p$ is strongly influenced by a compact invariant set $K$ if it has a neighborhood $U_{p}$ with the following property: for every neighborhood $V$ of $K$ there is a $T \geq 0$ such that for every $x \in U_{p}$ we have $x[T, \infty) \subset V$ or $x(-\infty,-T] \subset V$. We also say that the neighborhood $U_{p}$ is strongly influenced by $K$. There are, with obvious changes, similar definitions for the notions of a point and a neighborhood strongly attracted or strongly repelled by $K$. 
If $p$ is strongly influenced by $K$ then $p \in \mathcal{I}(K)$. Moreover, it is clear that if $p$ is strongly influenced by $K$ then all points in $\gamma(p)$ are strongly influenced by $K$.

Definition 10 provides all we need to characterize non-saddleness.

Proposition 11. The following are equivalent for an isolated invariant compactum $K$ :

i) $K$ is non-saddle;

ii) All points of $K$ are strongly influenced by $K$;

iii) $K$ has a neighborhood $U$ all whose points are strongly influenced by $K$.

Moreover, if $K$ is non-saddle then $\mathcal{I}(K)$ agrees with the set of all points strongly influenced by $K$, all points in $\mathcal{A}(K)-K$ are strongly attracted by $K$ and all points in $\mathcal{R}(K)-K$ are strongly repelled by $K$.

Proof. Conditions ii) and iii) are clearly equivalent since, by definition, strong influence on a point $p$ requires strong influence on all points of a neighborhood $U_{p}$. Moreover, if $K$ is non-saddle then every point in the interior of an isolating neighborhood of $K$ of the form $N=N^{+} \cup N^{-}$is strongly influenced by $K$ and, as a consequence, we have that i) implies iii). On the other hand suppose that all points of $K$ are strongly influenced by $K$. We claim that for every isolating neighborhood $N$ of $K$ there is an $\epsilon>0$ such that if a point $x \in N$ abandons $N$ in the past and in the future then $d(x, K)>\epsilon$. If not, there is and isolating neighborhood $N$ and a sequence of points $x_{n}$ contained in $N$ with $x_{n} \rightarrow x \in K$ such that every $x_{n}$ abandons $N$ in the past and in the future in times, say $T_{n}<0$ and $T_{n}^{\prime}>0$. Since $x_{n} \rightarrow x \in K$ and $K$ is invariant we must have that $T_{n} \rightarrow-\infty$ and $T_{n}^{\prime} \rightarrow \infty$. However, this is in contradiction with the fact that $x$ is strongly influenced by $K$. Hence such an $\epsilon>0$ exists. As a consequence, if we define $N_{0}=N^{+} \cup N^{-}$we obtain another isolating neighborhood $N_{0} \subset N$ with $N_{0}^{+}=N^{+}$and $N_{0}^{-}=N^{-}$and $K$ is non-saddle. Thus ii) implies i). Concerning the last assertion in the statement of the proposition, if $K$ is non-saddle and $x \in \mathcal{I}(K)$ then $\gamma(x)$ enters every isolating neighborhood of the form $N=N^{+} \cup N^{-}$, all whose points are strongly influenced by $K$. Hence $x$ is strongly influenced by $K$. If $x \in \mathcal{A}(K)-K$ then $\gamma^{+}(x)$ enters $N^{+}$and, hence $x$ is strongly attracted and similarly, if $x \in \mathcal{R}(K)-K$ then $x$ is strongly repelled.

\section{On the STRUCTURE OF A FLOW HAVING A NON-SADDLE SET}

This section is devoted to the study of the structure of a flow on a locally compact metric space having a non-saddle set. For this purpose we will use the following notation:

- $\mathcal{H}(K)=\mathcal{A}(K) \cap \mathcal{R}(K)$, the set of all points $x$ such that $\omega(x) \neq \emptyset, \omega^{*}(x) \neq \emptyset$ and $\omega(x) \cup \omega^{*}(x) \subset K$. If $x \in \mathcal{H}(K)-K$ we say that the point $x$ and the trajectory $\gamma(x)$ are homoclinic.

- $\mathcal{A}^{*}(K)=\mathcal{A}(K)-\mathcal{R}(K)$, the set of all points $x$ such that $\emptyset \neq \omega(x) \subset K$ but $\omega^{*}(x) \nsubseteq K$ or $\omega^{*}(x)=\emptyset$.

- $\mathcal{R}^{*}(K)=\mathcal{R}(K)-\mathcal{A}(K)$, the set of all points $x$ such that $\emptyset \neq \omega^{*}(x) \subset K$ but $\omega(x) \nsubseteq K$ or $\omega(x)=\emptyset$.

Proposition 12. Let $K$ be an isolated non-saddle set. Then:

i) $\mathcal{H}(K)-K$ is an open set in $M$.

ii) $\mathcal{A}^{*}(K) \cup K$ and $\mathcal{R}^{*}(K) \cup K$ are closed in $\mathcal{I}(K)$. 
Proof. If $x \in \mathcal{H}(K)-K$ then $x$ is both strongly attracted and strongly repelled by $K$, which means that it has a neighborhood $U_{x}$ contained in $\mathcal{A}(K) \cap \mathcal{R}(K)$ and not meeting $K$. Hence $\mathcal{H}(K)-K$ is open.

To prove ii) let us argue by contradiction. If $\mathcal{A}^{*}(K) \cup K$ is not closed in $\mathcal{I}(K)$ then there exists a sequence $x_{n} \rightarrow x \in \mathcal{I}(K)$ with $x_{n} \in \mathcal{A}^{*}(K) \cup K$ and $x \notin \mathcal{A}^{*}(K) \cup K$. Since $x \notin \mathcal{A}^{*}(K) \cup K$ we must have that $x \in \mathcal{R}(K)-K$ and, as a consequence, $x$ is strongly repelled by $K$. But this implies that $x_{n}$ is repelled by $K$ for almost all $n$, which is in contradiction with the choice of the sequence $x_{n}$.

In the sequel we will be concerned with the study of the region of influence $\mathcal{I}(K)$ and, in particular, with the structure of $\mathcal{I}(K)-K$. By the previous results, $\mathcal{I}(K)-K$ is the disjoint union of the sets $\mathcal{H}(K)-K, \mathcal{A}^{*}(K)$ and $\mathcal{R}^{*}(K)$ where $\mathcal{H}(K)-K$ is open and $\mathcal{A}^{*}(K)$ and $\mathcal{R}^{*}(K)$ are closed in $\mathcal{I}(K)-K$. However, $\mathcal{H}(K)-K$ is not necessarily closed in $\mathcal{I}(K)-K$ as the following example shows.

Example 13. This example shows an isolated non-saddle set $K$ of a flow on the torus $T$ (which is represented in Figure 2 as a square with opposite sides identified). All the points

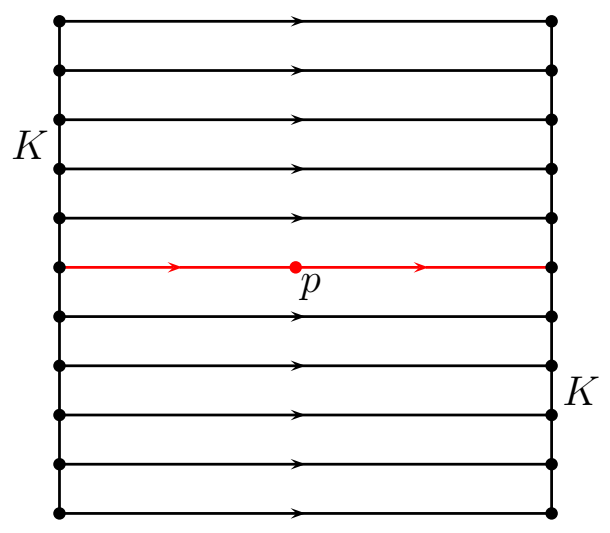

Figure 2. Flow on the torus

of $K$ are stationary and, in addition, there is a fixed point $p \notin K$. The orbits of all points $x \in T-K$ are homoclinic except the equilibrium $p$, the orbit finishing in $p$ and the orbit starting in $p$. The region of influence of $K$ is $\mathcal{I}(K)=T-\{p\}$. The set $\mathcal{H}(K)-K$ is not closed in $\mathcal{I}(K)-K$.

The fact that $\mathcal{H}(K)-K$ is not necessarily closed in $\mathcal{I}(K)-K$ (and, hence, $\mathcal{H}(K)$ is not necessarily closed in $\mathcal{I}(K)$ ) accounts for much of the complexity of the structure of $\mathcal{I}(K)$, specially when compared, for example, with the more simple case of unstable attractors without external explosions, where $\mathcal{H}(K)$ is indeed closed in $\mathcal{I}(K)=\mathcal{A}(K)$. In spite of this, some properties of that family of unstable attractors are shared by non-saddle sets. However, if we want to have some understanding of the structure of the region of influence of non-saddle sets we must acknowledge the existence of a special kind of orbits which are responsible for both the topological and the dynamical complexity. This we will do in Definition 15. 
In the sequel we will often use the prolongational limits $J^{+}, J^{-}$and $J^{*}$. The following result, whose proof is left to the reader, provides a useful characterization of attracting, repelling and homoclinic points lying outside $K$.

Proposition 14. Let $K$ be an isolated non-saddle set of a flow on $M$ and $x \in M-K$. Then

i) $x \in \mathcal{A}(K)$ if and only if $J^{+}(x) \neq \emptyset$ and $J^{+}(x) \subset K$.

ii) $x \in \mathcal{R}(K)$ if and only if $J^{-}(x) \neq \emptyset$ and $J^{-}(x) \subset K$.

iii) If $x \in \mathcal{H}(K)$ then $J^{*}(x) \neq \emptyset$ and $J^{*}(x) \subset K \times K$. The converse holds if $M$ is compact.

We stress that the previous proposition refers to points $x \in M-K$ only. These properties do not generally hold for points in $K$.

In the following definition we introduce a kind of points which play an essential role in our discussion.

Definition 15. A point $x \in \mathcal{I}(K)$ is said to be positively dissonant if $x \notin \mathcal{A}(K)$ (in which case $x \in \mathcal{R}(K))$ but $J^{+}(x) \cap K \neq \emptyset$. We also say that the orbit $\gamma(x)$ is positively dissonant. There is a similar definition for negatively dissonant points and orbits. A point $x \notin \mathcal{I}(K)$ and its orbit are said to be externally dissonant if $J^{*}(x) \cap(K \times K) \neq \emptyset$. We denote by $\mathcal{D}$ the set of all dissonant points .

This definition conveys the idea that positively dissonant points are not attracted by $K$ but, nevertheless, $K$ has a kind of attractive influence on some points close to them. Externally dissonant points do not belong to the region of influence of $K$ (therefore they are neither atracted nor repelled) but $K$ has simultaneously a kind of attractive and repulsive influence on some points close to them. We remark that a flow on $M$ migth have an isolated nonsaddle set $K$ and points $x$ in $M-\mathcal{I}(K)$ with $J^{+}(x) \cap K \neq \emptyset$ and $J^{-}(x) \cap K \neq \emptyset$ but $J^{*}(x) \cap(K \times K)=\emptyset$. Obviously, such points are not externally dissonant.

By using dissonant points we can give a nice dynamical characterization of the closure of the set of homoclinic points.

Proposition 16. Let $x$ be a point not contained in $K$. Then $x$ is dissonant if and only if it is in the boundary of $\mathcal{H}(K)$. As a consequence $\overline{\mathcal{H}(K)}=\mathcal{H}(K) \cup \mathcal{D}$ i.e. the closure or $\mathcal{H}(K)$ consists of $K$ and its homoclinic points together with the dissonant points of $K$.

Proof. Suppose $x$ is in the boundary of $\mathcal{H}(K)$. Then $x$ is the limit of a sequence of points $x_{n} \in \mathcal{H}(K)$, and hence there exist a subsequence $x_{n_{k}}$, sequences $t_{k} \rightarrow \infty$ and $s_{k} \rightarrow-\infty$ and points $y, z \in K$ with $x_{n_{k}} t_{k} \rightarrow y, x_{n_{k}} s_{k} \rightarrow z$. As a consequence, if $x \notin \mathcal{I}(K)$ then $x$ is an externally dissonant point. If $x \in \mathcal{I}(K)-K$ and $x \in \mathcal{A}(K)$ then $x \notin \mathcal{R}(K)$ since, otherwise, $x$ would be in $\mathcal{H}(K)-K$, which is an open set of $M$, and this is in contradiction with $x$ being in the boundary of $\mathcal{H}(K)$. Since $x \notin \mathcal{R}(K), x_{n_{k}} \rightarrow x$ and $x_{n_{k}} s_{k} \rightarrow z \in K$ we have that $x$ is a negatively dissonant point. An analogous argument applies when $x \in \mathcal{R}(K)$. This proves that $x$ is dissonant whenever $x$ is in the boundary of $\mathcal{H}(K)$ and $x \notin K$. On the other hand if $x \notin \mathcal{I}(K)$ is an externally dissonant point, there exist $x_{n} \rightarrow x, t_{n} \rightarrow \infty, s_{n} \rightarrow-\infty$ with $x_{n} t_{n} \rightarrow y$ and $x_{n} s_{n} \rightarrow z$ and $(y, z) \in K \times K$. Then the points $x_{n} \in \mathcal{H}(K)$ for all $n$ sufficiently large and, thus, $x$ is in the boundary of $\mathcal{H}(K)$. Positively and negatively dissonant points are readily seen to belong to the boundary of $\mathcal{H}(K)$. 
Remark 17. Observe that if $K$ does not have dissonant points and $\mathcal{H}(K)-K \neq \emptyset$, then $\mathcal{H}(K)$ is compact and hence an isolated non-saddle set. Moreover, $\mathcal{H}(K)$ is the smallest simple non-saddle set containing $K$ and its region of influence agrees with $\mathcal{I}(K)$.

The next result deepens into the topological structure of $\mathcal{I}(K)-K$.

Proposition 18. Let $K$ be an isolated non-saddle set of a flow $\varphi$ defined on a locally compact ANR $M$ and $N=N^{+} \cup N^{-}$an isolating block of $K$ contained in $\mathcal{I}(K)$. Then, $\mathcal{I}(K)-K$ has a finite number of connected components. Moreover, if a component $C$ does not contain dissonant points the restriction flow $\varphi \mid C$ is parallelizable with section a component of $\partial N$. Hence, the flow provides a strong deformation retraction of $C$ onto a component of $\partial N \cap C$. Conversely, if the restriction flow $\varphi \mid C$ to a component $C$ of $\mathcal{I}(K)-K$ is parallelizable, then $C$ does not contain dissonant points.

Proof. It is easy to see that $N$ lies in the interior of another isolating block $N_{0}=N_{0}^{+} \cup N_{0}^{-}$ and that there exists a retraction of $\stackrel{\circ}{0}_{0}-K$ onto $\partial N$ provided by the flow. As a consequence, $\partial N$ is an ANR and hence, it has a finite number of components. Since every component of $\mathcal{I}(K)-K$ contains at least one component of $\partial N$ there must be a finite number of them.

Suppose that $C$ is a component of $\mathcal{I}(K)-K$ which does not contain dissonant points. Then, by Proposition 12 and Proposition 16, $C$ is contained in one of the subsets $\mathcal{A}^{*}(K)$, $\mathcal{R}^{*}(K)$ or $\mathcal{H}(K)-K$ and, hence, $K$ is either a global attractor or a global repeller or a global unstable attractor with no external explosions respectively for the restriction flow $\varphi \mid C \cup K$. Then, by [5] and [33] the flow in $C$ is parallelizable with section a component of $\partial N$.

Conversely, assume that $\varphi \mid C$ is parallelizable. We see that $C$ does not contain dissonant points. Choose an isolating block $N=N^{+} \cup N^{-}$of $K$. Suppose, arguing by contradiction, that $x \in C$ is a positively dissonant point the other case being analogous. Then there exists a sequence of homoclinic points $x_{n} \in C$ such that $x_{n} \rightarrow x$. For each $n$ choose $t_{n}$ to be the unique time for which $x_{n} t_{n} \in N^{i} \cap C$. Since $N^{i} \cap C$ is compact we may assume that $x_{n} t_{n} \rightarrow y \in N^{i} \cap C$. Besides, since $x$ is positively dissonant then $x \notin \mathcal{A}(K)$ and, as a consequence, $t_{n} \rightarrow+\infty$ in contradiction with $\varphi \mid C$ being parallelizable.

Remark 19. For the second part of Proposition 18 there is no need of $M$ to be an ANR. It also holds if $M$ is a locally compact metric space.

The existence of positively, negatively and externally dissonant points is mutually related as the following result shows.

Proposition 20. If an isolated non-saddle set $K$ has externally dissonant points, then it has also positively and negatively dissonant points. Conversely, if $M$ is compact and $K$ has either positively or negatively dissonant points in $\mathcal{I}(K)$ then $K$ has externally dissonant points.

Proof. Suppose that $x$ is an externally dissonant point. Then there exists a sequence $x_{n}$ in $\mathcal{H}(K)-K$ such that $x_{n} \rightarrow x$. Let $N=N^{+} \cup N^{-}$be an isolating block of $K$ (which must be contained in $\mathcal{I}(K))$. For almost all $n$ there exists $t_{n}>0$ such that $x_{n} t_{n} \in N^{i}$. By compactness we may assume that $x_{n} t_{n} \rightarrow y \in N^{i} \subset \mathcal{A}(K)-K$. The sequence $t_{n} \rightarrow+\infty$ since, otherwise it would have a bounded subsequence $t_{n_{k}}$ such that $t_{n_{k}} \rightarrow t_{0}$. Then, $x_{n_{k}} t_{n_{k}} \rightarrow y=x t_{0}$ which is a contradiction since $x t_{0} \notin \mathcal{I}(K)$. As a consequence $y \in J^{+}(x)$ and by [5] $x \in J^{-}(y)$. Then 
$J^{-}(y) \cap K \neq \emptyset$ but $J^{-}(y) \nsubseteq K$ and, hence, $y$ is a negatively dissonant point. An analogous argument leads us to find a positively dissonant point.

For the converse, suppose that $M$ is compact and there exists a negatively dissonant point $x$. Then there exists a sequence $x_{n}$ in $\mathcal{H}(K)-K$ such that $x_{n} \rightarrow x$. By assumption, $\emptyset \neq \omega^{*}(x) \subset M-\mathcal{I}(K)$. Let $y \in \omega^{*}(x)$. Then there exists a sequence $t_{n} \rightarrow-\infty$ such that $x t_{n} \rightarrow y$. Given $\epsilon>0$, it is possible to choose subsequences $x_{n_{k}}$ and $t_{n_{k}}$ such that $d\left(x_{n_{k}} t_{n_{k}}, x t_{n_{k}}\right)<\epsilon / 2$ for $k$ larger than a certain $k_{0}$. Moreover, there exists $k_{1}$ such that if $k \geq k_{1}$, then $d\left(x t_{n_{k}}, y\right)<\epsilon / 2$. As a consequence $d\left(x_{n_{k}} t_{n_{k}}, y\right) \leq d\left(x_{n_{k}} t_{n_{k}}, x t_{n_{k}}\right)+d\left(x t_{n_{k}}, y\right)<\epsilon$, for $k \geq \max \left\{k_{0}, k_{1}\right\}$. This proves that the point $y$ is externally dissonant. If the point $x$ is chosen to be positively dissonant the argument is analogous.

Remark 21. In the previous proposition it is proved that points $x \notin \mathcal{I}(K)$ which are $\omega^{*}$ limits of negatively dissonant points are externally dissonant, and the same is true for $\omega$-limits of positively dissonant points, but it can be easily shown that the converse does not hold. In fact, there are externally dissonant points which are neither $\omega$-limits nor $\omega^{*}$-limits of points of $\mathcal{I}(K)$. However, in the same proposition it is proved the weaker property that externally dissonant points lie in the positive prolongational limit $\left(\mathrm{J}^{+}\right)$of positively dissonant points and in the negative prolongational limit $\left(J^{-}\right)$of negatively dissonant points.

Example 22. Our previous Example 13 can be presented in a more general way. Consider the vector field in the torus induced by the differential equation in the square $I^{2}=[0,1]^{2} \subset \mathbb{R}^{2}$ defined by

$$
\left\{\begin{array}{l}
\dot{x}=\psi(x)\left(x^{2}+y^{2}-\frac{1}{4}\right) \\
\dot{y}=0
\end{array}\right.
$$

Where $\psi:[-1,1] \rightarrow[0,1]$ is a smooth function satisfying that $\psi(x)=1$ if $x \in[-1 / 2,1 / 2]$ and $\psi(x)=0$ if and only if $x= \pm 1$.

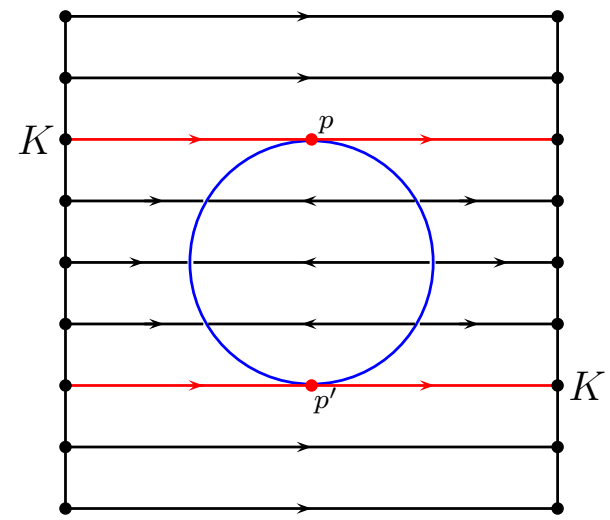

Figure 3. Saddle-node bifurcations at $p$ and $p^{\prime}$

The phase diagram of this flow can be understood as a collective phase diagram of a saddlenode bifurcation at the point $p$ (and symetrically at the point $p^{\prime}$ ). All points contained in the blue circle are fixed. The bifurcation points agree in this example with the externally dissonant points. The boundary of the region of influence of $K$ separates the torus into two connected components. 
We will see that the previous example is only a particular instance of a much more general situation.

Sánchez-Gabites proved in [33] a topological result which he used to construct families of unstable attractors in manifolds and which is useful as well in the present context. The proof of the result makes use of a classical theorem by Thom [40] about representation of homology classes. We reproduce its statement here because, originally, this result is contained in the proof of a different proposition and it is not formulated separately.

Proposition 23. (Sánchez-Gabites). Let $M$ be a connected oriented closed smooth n-manifold with $H^{1}(M) \neq\{0\}$ (or, equivalently, with $H_{n-1}(M) \neq\{0\}$ ). Then there exists a connected oriented closed smooth hypersurface $Z \subset M$ satisfying the following conditions:

i) $Z$ has a product neighborhood in $M$, i.e. there exists an open neighborhood $U$ and a homeomorphism $h: Z \times \mathbb{R} \rightarrow U$ with $h(z, 0)=z$ for every $z \in Z$.

ii) $M-U$ is connected.

It turns out that Proposition 23 is useful to construct non-saddle sets. The next result illustrates that non-saddle sets with dissonant points are indeed abundant. Its proof is, simply, an adaptation of the argument given in [33] for unstable attractors without external explosions.

Theorem 24. Let $M$ be a connected closed oriented smooth $n$-manifold with $n \geq 2$. If $H^{1}(M) \neq\{0\}$ then there exists a flow on $M$ having a connected isolated non-saddle set with dissonant points.

Proof. We use the notation of Proposition 23. Construct a flow on $Z \times \mathbb{R}$ such that $Z \times(-\infty, 0]$ and $Z \times[1, \infty)$ consist of fixed points and points in $Z \times(0,1)$ move from $Z \times\{0\}$ to $Z \times\{1\}$ except for a distinguished point $z_{0}$ for which the interval $\left\{z_{0}\right\} \times(-1,1)$ is broken in three different trajectories covering $\left\{z_{0}\right\} \times(-1,0),\left\{z_{0}\right\} \times\{0\}$ and $\left\{z_{0}\right\} \times(0,1)$ respectively. Now carry this flow to $U$ via $h$ and extend it to $M$ by leaving fixed all points in $M-U$. It is easy to see that $K=M-U$ is an isolated non-saddle set, $h\left(\left\{z_{0}\right\} \times(-1,0)\right)$ and $h\left(\left\{z_{0}\right\} \times(0,1)\right)$ are positively and negatively dissonant orbits and $h\left(z_{0}\right)$ is an externally dissonant point.

Theorem 24applies to manifolds with $H^{1}(M) \neq\{0\}$. However, for manifolds with $H^{1}(M)=$ $\{0\}$ the situation is much simpler.

Theorem 25. Let $M$ be a connected closed manifold with $H^{1}(M)=\{0\}$ and suppose that $K$ is a connected isolated non-saddle set of a flow on $M$. Then, $K$ does not have dissonant points. Moreover, if $U$ is a component of $M-K$ then the flow restricted to $U$ is either locally attracted by $K$ (i.e. all points lying in $U$ near $K$ are attracted by $K$ ) or locally repelled by $K$. Furthermore, if $N$ is an isolating block of $K$ of the form $N=N^{+} \cup N^{-}$then different components of $\partial N$ lie in different components of $M-K$. As a consequence, connected isolated non-saddle sets $K$ of flows in the Euclidean space $\mathbb{R}^{n}, n \geq 2$, have no dissonant points and the flow on every component of $\mathbb{R}^{n}-K$ is either locally attracted or locally repelled by $K$. In particular, if $\mathcal{I}(K)=\mathbb{R}^{n}$ then $K$ is a stable global attractor or a negatively stable global repeller.

Proof. Since $K$ is isolated then $M-K$ consists of a finite number of components. We will study the behaviour of the flow on every component of $M-K$. Suppose $U$ is one of such 
components. Let $\mathscr{C}$ be the set whose elements are all the components of $M-K$ different from $U$. We define

$$
\hat{K}=K \bigcup\left(\bigcup_{V \in \mathscr{C}} V\right)
$$

Then $\hat{K}$ is a connected isolated invariant non-saddle set of the flow with $M-\hat{K}=U$. Consider an isolating block $N$ of $\hat{K}$ such that $N=N^{+} \cup N^{-}$. We will prove that $\hat{K}$ does not disconnect $\stackrel{\circ}{N}$. By considering the initial part of the long exact cohomology sequence of the pair $(M, M-\hat{K})$ we obtain

$$
\ldots \leftarrow H^{1}(M)=\{0\} \leftarrow H^{1}(M, M-\hat{K}) \leftarrow \widetilde{H}^{0}(M-\hat{K})=\{0\} \leftarrow \widetilde{H}^{0}(M)
$$

and, as a consequence, $H^{1}(M, M-\hat{K})=\{0\}$. By excising from $M$ the compact set $L=M-\stackrel{\circ}{N}$ we get $H^{1}(\stackrel{\circ}{N}, \stackrel{\circ}{N}-\hat{K})=H^{1}(M-L,(M-L)-\hat{K}) \cong H^{1}(M, M-\hat{K})=\{0\}$. Hence, from the the initial part of the long exact cohomology sequence of the pair $(\stackrel{\circ}{N}, \stackrel{\circ}{N}-\hat{K})$ we get

$$
\ldots \leftarrow H^{1}(\stackrel{\circ}{N}, \stackrel{\circ}{N}-\hat{K})=\{0\} \leftarrow \widetilde{H}^{0}(\stackrel{\circ}{N}-\hat{K}) \leftarrow \widetilde{H}^{0}(\stackrel{\circ}{N})=\{0\}
$$

and, as a consequence, $\widetilde{H}^{0}(\stackrel{\circ}{N}-\hat{K})=\{0\}$ which proves that $\hat{K}$ does not disconnect $\stackrel{\circ}{N}$. Hence, since $N$ can be chosen to be arbitrarily small, Proposition 7 ensures that either $\hat{K}$ is an attractor in which case all points of $N$ in $U$ are attracted to $K$ or $\hat{K}$ is a repeller, in which case all points of $N$ in $U$ are repelled by $K$. On the other hand, a similar argument proves that different components of $\partial N$ lie in different components of $M-K$.

Moreover $K$ has no homoclinic orbits and thus no dissonant points. The claim about $\mathbb{R}^{n}$ is readily obtained by considering its Alexandrov compactification i.e. the $n$-dimensional sphere $S^{n}$ and applying to $S^{n}$ the previous part of the theorem.

A nice consequence in the case of unstable attractors in $\mathbb{R}^{n}$ is the following result from [25].

Corollary 26. All connected isolated unstable attractors of flows on $\mathbb{R}^{n}$ have external explosions.

The next theorem gives a local sufficient condition for an isolated invariant continuum to be non-saddle in terms of its Conley index.

Theorem 27. Let $M$ be a connected and locally compact metric space and suppose that $K$ is an isolated invariant continuum of a flow $\varphi$ on $M$ such that $K$ disconnects a connected neighborhood $W$ of $K$ in $M$ into two components. If $C H_{+}^{1}(K)$ and $C H_{-}^{1}(K)$ are trivial then $K$ is non-saddle and it is neither an attractor nor a repeller. Moreover, if $M$ is a compact manifold without boundary such that $K$ does not disconnect $M$ then $H^{1}(M) \neq\{0\}$.

Proof. Let $W$ be a connected neighborhood of $K$ in $M$ such that $W-K$ consists of two different connected components, $C_{1}$ and $C_{2}$. Consider a connected isolating block $N$ of $K$ contained in $W$ with entrance and exist sets $N^{i}$ and $N^{o}$ respectively. We remark that if $N^{o}$ is empty then $N^{i}$ is necessarily non-empty and consisting of at least two components (one at least in each $C_{i}$ ). Suppose that $N^{o}$ is non-empty. Since $C H_{+}^{1}(K)=\{0\}$ we have that $\check{H}^{1}\left(N, N^{o}\right)$ is trivial and from the cohomology exact sequence of the pair $\left(N, N^{o}\right)$

$$
\ldots \leftarrow \check{H}^{1}\left(N, N^{o}\right)=\{0\} \leftarrow \widetilde{\check{H}^{0}}\left(N^{o}\right) \leftarrow \widetilde{\check{H}^{0}}(N)=\{0\}
$$


we get that $\widetilde{\breve{H}^{0}}\left(N^{o}\right)=\{0\}$ and, as a consequence $N^{o}$ is connected. A similar argument using the fact that $C H_{-}^{1}(K)$ is trivial would establish that if $N^{i}$ is non-empty then it is also connected. From this, we get that if both $N^{i}$ and $N^{o}$ are non-empty then the boundary $\partial N$ consists of exactly two connected components $N^{o}$ and $N^{i}$ with one of them $N^{o}$ contained in, say, $C_{1}$, and the other, $N^{i}$, contained in $C_{2}$. The previous argument can be used to exclude the fact that one of the sets $N^{i}$ or $N^{o}$ is empty since, in that case, the other would be nonempty and consisting of more than one component and we would have a contradiction. Then $K$ can be neither an attractor nor a repeller and it readily follows that $N$ is an isolating block for $K$ with the structure $N=N^{+} \cup N^{-}$and, thus, that $K$ is non-saddle. On the other and, if $M$ is a manifold not disconnected by $K$ and $H^{1}(M)=\{0\}$ then, by Theorem 25, $K$ attracts or repells all the points of one of its neighborhoods in $M$ and this is a contradiction with the fact just proved that $K$ is neither an attractor nor a repeller.

The next example is a modification of [25, Example 2] which shows how to produce plenty of examples of flows having an isolated invariant continuum in the conditions of Theorem 27.

Example 28. Let $K$ be a compact and connected manifold (without boundary) endowed with a flow $\varphi_{1}$ and consider the unit interval $[0,1]$ together with a dynamical system $\varphi_{2}$ which has 0 and 1 as fixed points and otherwise moves points away from 0 and towards 1 . The product flow $\varphi(x, s, t):=\left(\varphi_{1}(x, t), \varphi_{2}(s, t)\right)$ in the phase space $K \times[0,1]$ restricts to $\varphi_{1}$ on $K \times\{0\}$ and $K \times\{1\}$, hence these can be identified to get a flow on the quotient space $K \times S^{1}$ (with the obvious identifications). Observe that given a closed product neighborhood $W$ of $K$, i.e. a neighborhood mapped onto $K \times[-1,1]$ by a homeomorphism $h: W \rightarrow K \times[-1,1]$ such that $h(K)=K \times\{0\}, W$ is disconnected by $K$ into two components. Moreover, $W$ can be chosen to be an isolating block with entrance and exit set corresponding to $K \times\{-1\}$ and $K \times\{1\}$ respectively. Then, $C H_{+}^{1}(K)=C H_{-}^{1}(K)=\{0\}$ and Theorem 27 ensures that $K$ is non-saddle. A flow $\widehat{\varphi}$ in the conditions of Theorem 27 having dissonant points can be obtained modifying $\varphi$. Indeed, let $S$ be a proper closed subset of the exit set of $W$. By using a theorem of Beck [3] $\varphi$ can be modified to a new flow in such a way that all the orbits of $\varphi$ not meeting a point of $S$ are preserved while the orbits containing a point of $S$ are decomposed in two orbits together with that point of $S$. After this modification $W$ is no longer an isolating block but it is possible to build isolating blocks of $K$ as before in the interior of $W$. It is straightforward to see that $S$ contains externally dissonant points.

We will study now the general structure of a flow on a compact ANR having an isolated non-saddle set. The next result gives an overall picture of the situation.

Theorem 29. Let $K$ be a connected isolated non-saddle set of a flow on a compact and connected ANR, M, (in particular on a compact and connected manifold) and let $L$ be the complement $M-\mathcal{I}(K)$ of its region of influence. Then $L$ is an isolated invariant compactum which is non-empty when $K$ is not an unstable global attractor. The saddle components of $L$ are exactly those containing externally dissonant points of $K$. The union of these components is an isolated invariant (saddle) compactum $L_{s}$ and $L-L_{s}$ is an isolated invariant non-saddle compactum that we denote by $L_{n}$. Moreover, if $x$ is a non-homoclinic point in $\mathcal{I}(K)-K$ and the component of $\mathcal{I}(K)-K$ containing $x$ contains also homoclinic points then either $\omega(x) \subset L_{s}$ or $\omega^{*}(x) \subset L_{s}$. 
Proof. Obviously, $L$ is compact and invariant being the complement in $M$ of the open invariant set $\mathcal{I}(K)$ and it is non-empty when $K$ is not an unstable global attractor. Suppose that $L$ is non-empty since otherwise there is nothing to prove. Then, if $U$ is a closed neighborhood of $L$ with $U \cap K=\emptyset$ then the trajectory of every point in $U-L$ is contained in $\mathcal{I}(K)$ and hence either its $\omega$-limit or its $\omega^{*}$-limit is in $K$, which implies that this trajectory is not entirely contained in $U$. Hence $L$ is isolated.

Suppose that $C$ is a component of $L$. We see that $C$ contains externally dissonant points if and only if $C$ is saddle. Suppose that $C$ is saddle. Then there exists a neighborhood $U$ of $C$ disjoint from $K$ and a sequence of points $x_{n} \in U, x_{n} \rightarrow C$ such that $\gamma^{+}\left(x_{n}\right) \nsubseteq U$ and $\gamma^{-}\left(x_{n}\right) \nsubseteq U$. Choose an isolating block $N$ of $L$ such that the component $N_{C}$ of $N$ containing $C$ is contained in $U$. Since for each $n, x_{n}$ belongs to $\mathcal{I}(K)$ we may assume without loss of generality that either $\omega\left(x_{n}\right) \subset K$ for each $n$ or $\omega^{*}\left(x_{n}\right) \subset K$ for each $n$. We consider the first situation. The trajectory of $x_{n}$ abandons $N_{C}$ in positive and negative time since $N_{C} \subset U$. Let $y_{n}$ be the sequence corresponding to the exit points of $\gamma^{+}\left(x_{n}\right)$. We may assume that $y_{n} \rightarrow y \in \partial N_{C}$ and a simple argument shows that $\omega^{*}(y) \subset L$. As a consequence $\omega^{*}\left(x_{n}\right) \subset K$ for almost all $n$ since if not, there is a contradiction with the fact that $\mathcal{A}^{*}(K)$ is closed in $\mathcal{I}(K)-K$. This proves that $C$ contains externally dissonant points. The converse statement is straightforward and it is left to the reader.

We see that $L_{s}$ is a compactum. Otherwise there are points $x_{n}$ in $L_{s}$ converging to a point $x \in L_{n}$ and hence $x$ belongs to a non-saddle component $C$ of $M-\mathcal{I}(K)$. Consider an arbitrary neighborhood $U$ of $C$ not meeting $K$. Then there is an arbitrarily small neighborhood $U^{\prime} \subset U$ of $C$ such that if a component of $L$ meets $U^{\prime}$ then it is entirely contained in $U^{\prime}$. Hence $U^{\prime}$ contains the components of some $x_{n}$ and, as a consequence, dissonant and, thus, homoclinic points whose orbit leaves $U$ in the past and in the future. Hence $C$ cannot be non-saddle.

We see now that every component $C$ of $L_{n}$ is isolated. Consider a closed neighborhood $U$ of $L$ such that the component $U_{0}$ containing $C$ does not meet $L_{s} \cup K$. Then $U_{0} \cap L$ is a compactum and $U_{0}$ can be chosen in such a way that $U_{\cap} L$ consists entirely of non-saddle components. Hence $U_{0} \cap L$ is non-saddle. Moreover $U_{0} \cap L$ is isolated, hence it consists of a finite number of components all isolated non-saddle. On the other hand, we will prove that $L_{n}$ itself consists of a finite number of components. Consider an isolating block $N$ of $K$ in $M$. We know that its boundary, $\partial N$, consists of a finite number of components. Let $C$ be a component of $L_{n}$ and $S$ the set of points of $\partial N$ which are attracted or repelled by $C$. We will see that $S$ is open-closed in $\partial N$ and, hence, $S$ attracts or repells complete components of $\partial N$. We prove that $S$ is a closed subset of $\partial N$. Suppose $x_{n} \in S$ are attracted by $C$ and $x_{n} \rightarrow x$. All the points $x_{n}$ and $x$ itself belong to $\mathcal{R}^{*}(K)$. If $x$ is not attracted by $C$ then $\omega(x)$ is contained in a component $C^{\prime} \subset L_{s}$. Consider an isolating block $N$ of $L$ such that $C$ and $C^{\prime}$ lie in different components $N_{C}$ and $N_{C^{\prime}}$ of $N$. We have points $x_{n} s_{n} \in N_{C^{\prime}}^{o}$ with $s_{n} \rightarrow \infty$. The points $x_{n} s_{n}$ converge to $y \in N_{C^{\prime}}^{o}$ with $y s \in N_{C^{\prime}}$ for every negative $s$. Hence $\omega^{*}(y) \subset N_{C^{\prime}}$ and $y \notin \mathcal{R}(K)$. Hence $\mathcal{R}^{*}(K)$ is not closed in $\mathcal{I}(K)-K$. This contradiction proves that $S$ is open-closed in $\partial N$ and hence $C$ attracts or repells complete components. Since every $C$ must attract or repell some component we deduce that there is a finite number of non-saddle components and, thus, $L_{n}$ is isolated non-saddle. 
If the component of $\mathcal{I}(K)-K$ containing $x$ contains homoclinic points then the component of $\partial N$ containing its orbit contains homoclinic points and by the previous discussion it can be neither attracted nor reppelled by $L_{n}$. Then, either $\omega(x) \subset L_{s}$ or $\omega^{*}(x) \subset L_{s}$.

Corollary 30. $M-\mathcal{I}(K)$ is non-saddle if and only if the flow does not have dissonant points.

We say that an orbit $\gamma \subset \mathcal{I}(K)-K$ is deviant if either $\omega(\gamma)$ or $\omega^{*}(\gamma)$ contains an externally dissonant point.

All the externally dissonant points are contained in $L_{s}$. In the important particular case in which all the points of $L_{s}$ are dissonant we have

Corollary 31. If $L_{s}$ consists entirely of dissonant points then $\hat{K}=\mathcal{H}(K) \cup \mathcal{I}\left(L_{s}\right)$ is the smallest simple non-saddle set containing $K$ i.e. $\hat{K}$ is obtained from $K$ by adding all the homoclinic orbits plus all the deviant orbits.

\section{NON-SADDLE SETS In 2-DIMENSIONAL FLOWS}

In this section we will study some dynamical properties of non-saddle sets of flows defined on 2-dimensional manifolds. In particular, the following result shows that in the case of closed orientable surfaces, the existence of dissonant points can be detected by looking at some relations between the topologies of $K$ and its region of influence. Since in this section we are dealing with either 2-dimensional manifolds or the Euclidean plane $\mathbb{R}^{2}$, Gutierrez' Theorem allows us to assume that all the flows considered are $C^{1}$ and $C^{\infty}$ respectively.

Theorem 32. An isolated non-saddle continuum $K$ of a flow on a closed orientable surface $M$ does not have dissonant points if and only if the homology and cohomology of $\mathcal{I}(K)$ are finitely generated and $\chi(K)=\chi(\mathcal{I}(K))$.

Proof. Suppose that $K$ has no dissonant points. It follows from Alexander duality theorem that

$$
H_{k}(\mathcal{I}(K), \mathcal{I}(K)-K) \cong \check{H}^{2-k}(K)
$$

and, since $K$ has finitely generated Čech cohomology, $\chi(\mathcal{I}(K), \mathcal{I}(K)-K)$ is defined and agrees with $\chi(K)$. Moreover, if $K$ does not have dissonant points then $\mathcal{I}(K)$ and $\mathcal{H}(K)$ are of the same shape, which implies, since $\mathcal{H}(K)$ is non-saddle, that $\chi(\mathcal{I}(K))$ is well defined. As a consequence, $\chi(\mathcal{I}(K)-K)$ is also defined and

$$
\begin{array}{r}
\chi(\mathcal{I}(K))=\chi(\mathcal{I}(K), \mathcal{I}(K)-K)+\chi(\mathcal{I}(K)-K)= \\
\chi(K)+\chi(\mathcal{I}(K)-K) .
\end{array}
$$

Consider now an isolating block $N$ of $K$ in $M$ of the form $N=N^{+} \cup N^{-}$. We may suppose by [9] that $N$ is a compact surface whose boundary $\partial N$ consists of a finite union of circles. Then there exists a strong deformation retraction of $\mathcal{I}(K)-K$ onto some of those circles (especifically onto the union of $N^{o}$ with $\left.N^{i}-\mathcal{H}(K)\right)$ and it follows that $\chi(\mathcal{I}(K)-K)=0$. As a consequence, $\chi(\mathcal{I}(K))=\chi(K)$.

Conversely, suppose that the Euler characteristic of $\mathcal{I}(K)$ is defined and $\chi(K)=\chi(\mathcal{I}(K))$. Then $\chi(\mathcal{I}(K)-K)=0$. Moreover, $\mathcal{I}(K)-K$ is a disjoint union of connected orientable surfaces $S_{1}, \ldots, S_{n}$, all of them proper open subsets of the closed surface $M$. If $S_{i}$ does not contain dissonant points then there exists a strong deformation retraction of $S_{i}$ onto a 
component of $\partial N \cap S_{i}$ (a circle) and, thus, $\chi\left(S_{i}\right)=0$. Now suppose, to get a contradiction, that $S_{i}$ contains dissonant points. Since it is a proper subset of $M$ then $\chi\left(S_{i}\right) \leq 1$. We will see that the possibilities $\chi\left(S_{i}\right)=0$ or 1 are excluded. If $\chi\left(S_{i}\right)=0$ then by [28] $S_{i}$ is homeomorphic to a punctured open disk $D-\{p\}$. If $C$ is a component of $\partial N \cap S_{i}$ then $C$ is a circle not contractible in $S_{i}$ (otherwise the disc bounded by $C$ would be positively or negatively invariant by the flow and would contain an invariant set not influenced by $K$ ). The intersection $\partial N \cap S_{i}$ consists of more than one component since, otherwise, there would be no homoclinic orbits in $S_{i}$ and, thus, no dissonant points. The external and the internal components of $\partial N \cap S_{i}$ limit a region $R$ in $D-\{p\}$ homeomorphic to a planar ring. All the trajectories of points of $R$ abandon $R$ in the past and in the future (otherwise there would exist an invariant set in $R$ not influenced by $K)$. This implies that all the trajectories in $S_{i}$ are homoclinic and, hence, there are no dissonant points. A similar but easier argument excludes the possibility that $\chi\left(S_{i}\right)=1$. Hence $\chi\left(S_{i}\right)<0$ for every surface containing dissonant points. Since $\chi(\mathcal{I}(K)-K)=\sum_{i=1}^{n} \chi\left(S_{i}\right)=0$ and all the surfaces $S_{i}$ without dissonant points are of zero Euler characteristic, we conclude that there are no dissonant points in $\mathcal{I}(K)-K$.

The next result establishes a relation between the homoclinic orbits of a plane continuum and the existence of fixed points in its complement.

Lemma 33. Suppose that $K$ is an isolated invariant continuum of a plane flow and $\mathcal{H}(K)-$ $K \neq \emptyset$. Then there exists a fixed point in $\mathbb{R}^{2}-K$. More generally, if $K$ is an isolated invariant continuum of a plane flow and a component $U$ of $\mathbb{R}^{2}-K$ contains a trajectory $\gamma$ such that $\omega(\gamma) \cap K \neq \emptyset$ and $\omega^{*}(\gamma) \cap K \neq \emptyset$ then there exists a fixed point in $U$.

Proof. Suppose, to get a contradiction, that there exists a trajectory $\gamma$ in a component $U$ of $\mathbb{R}^{2}-K$ such that $U$ does not contain fixed points and $\omega(\gamma) \cap K \neq \emptyset$ and $\omega^{*}(\gamma) \cap K \neq \emptyset$. Take an isolating block $N$ of $K$. By [9] $N$ can be chosen to be a topological closed disk with $i$ holes, one for every bounded component of $\mathbb{R}^{2}-K$. We suppose that $U$ is the unbounded component (the argument being only slightly diferent in the other case) and consider the only circle $C \subset \partial N$ contained in $U$. Then there exists a point $x \in C \cap \gamma$ leaving $N$ and returning to $N$ after a time $t \neq 0$, i.e. such that $x t \in C$ and $x(0, t) \cap N=\emptyset$. The possibility that the time $t$ be positive or negative is irrelevant in this construction. Consider the $\operatorname{arc} A$ in $C$ with extremes $x$ and $x t$ such that the topological circle $x[0, t] \cup A$ does not contain $K$ in its interior. This arc can be mapped to the unit interval $I=[0,1]$ of the real line by a homeomorphism $h: A \rightarrow I$. If we take the point $x_{1} \in \AA$ corresponding to the center of $I$ then $x_{1}$ must leave $N$ (in the past or in the future) and return again since, otherwise, the Theorem of Poincaré-Bendixson would imply the existence of a fixed point in the disk limited by $x[0, t] \cup A$. Hence we can repeat the operation with $x_{1}\left[0, t_{1}\right] \cup A_{1}$, where $A_{1}$ is an arc in $A$ with extremes $x_{1}$ and $x_{1} t_{1}$ and the topological circle $x_{1}\left[0, t_{1}\right] \cup A_{1}$ does not contain $K$ in its interior. Now take $x_{2} \in \AA_{1}$ corresponding to the middle point of $h\left(A_{1}\right)$ and repeat the construction. In this way we obtain a sequence $A \supset A_{1} \supset A_{2} \supset \ldots$ of arcs whose intersection $\bigcap_{i=1}^{\infty} A_{i}$ consists of one point $p \in \partial N$. The orbit of $p$ defines an internal tangency to $\partial N$, which is in contradiction with the properties of isolating blocks. This contradiction proves that if $\omega(\gamma) \cap K \neq \emptyset$ and $\omega^{*}(\gamma) \cap K \neq \emptyset$ then there exists a fixed point in $U$. 
As a consequence of the last proposition a lower bound for the number of fixed points in the complement of an isolated invariant plane continuum is obtained.

Corollary 34. Let $K$ be an isolated invariant continuum of a plane flow. Suppose that $\mathbb{R}^{2}$ $-K$ has $i$ connected components. Then, there are at least $i-1$ fixed points in $\mathbb{R}^{2}-K$.

Proof. We see that, in fact, there is at least one fixed point in every bounded component $U$ of $\mathbb{R}^{2}-K$. Otherwise, if $\gamma$ is a trajectory in the bounded component $U$, where $U$ does not contain fixed points, then by Lemma 33 either $\omega(\gamma) \cap K=\emptyset$ or $\omega^{*}(\gamma) \cap K=\emptyset$. Hence, Poincaré-Bendixson Theorem ensures the existence of a periodic orbit contained in $U$, and thus the existence of a fixed point in its interior. This leads to a contradiction with the assumption, since the interior of any periodic orbit contained in $U$ is also contained in $U$. The number of bounded components of $\mathbb{R}^{2}-K$ is exactly $i-1$, so this contradiction establishes the corollary.

In [2] it was proved that the non-existence of fixed points in an isolated invariant continuum $K$ of a planar flow is a sufficient condition for $K$ to be non-saddle. We see in the next result that an assumption about the region of influence of an isolated invariant plane continuum is also sufficient to guarantee its non-saddleness.

Theorem 35. Let $K$ be an isolated invariant continuum of a flow on $\mathbb{R}^{2}$ and suppose that $\mathcal{I}(K)=\mathbb{R}^{2}$. Then $K$ is non-saddle and, as a consequence, a stable global attractor or $a$ negatively stable global repeller.

Proof. Suppose, to get a contradiction, that $K$ is saddle. Then there exist an isolating block $N$ which, by the previous corollary can be taken to be a topological closed disk, and a sequence of points $x_{n} \rightarrow K$ whose trajectories leave $N$ in the future and in the past. Denote by $y_{n}$ and $z_{n}$ the corrresponding exit points (in the future and in the past respectively). We may assume that $y_{n} \rightarrow y_{0} \in \partial N$ and $z_{n} \rightarrow z_{0} \in \partial N$. A simple argument proves that $\emptyset \neq \omega^{*}\left(y_{0}\right) \subset K$ and $\emptyset \neq \omega\left(z_{0}\right) \subset K$. Since $\mathcal{I}(K)=\mathbb{R}^{2}$ then we may suppose that either $\emptyset \neq \omega\left(y_{n}\right) \subset K$ for almost all $n$ or $\emptyset \neq \omega^{*}\left(y_{n}\right) \subset K$ for almost all $n$; we consider the first situation and suppose, for the sake of simplicity in notation, that $\emptyset \neq \omega\left(y_{n}\right) \subset K$ for $n \geq 1$. We may also assume that all points $y_{n}$ and $y_{0}$ are contained in an arc $A \subset N^{o}$ with no tangency points. Consider now an arbitrary isolating block $N_{1} \subset \stackrel{\circ}{N}$ which is also a disk. We can define a topological circle $C$, not having $K$ in its interior, consisting of the union of the following sets: a) the trajectory $\gamma^{-}\left(y_{0}\right)$ until it reaches $\partial N_{1}$ in a point $\left.a, \mathrm{~b}\right)$ an $\operatorname{arc} J \subset A \subset N^{o}$, linking $y_{0}$ to $y_{1}$, c) the trajectory $\gamma^{+}\left(y_{1}\right)$ until it reaches $\partial N_{1}$ in a point $b$ and d) an arc in $\partial N_{1}$ linking $a$ to $b$. Denote by $D$ the disk bounded by $C$.

Then $\stackrel{\circ}{J}$ is either an exit set or an entrance set for $D$. If $\dot{J}^{\circ}$ is an exit set then either $\gamma^{-}\left(y_{1}\right)$ is contained in $D$ or there exists a point of $\gamma^{-}\left(y_{1}\right)$ contained in $\partial N_{1}$. It is not difficult to see, using the Poincaré Bendixson Theorem that the first case is impossible. If $\stackrel{\circ}{J}^{\circ}$ is an entrance set for $D$ then either $\gamma^{+}\left(y_{0}\right)$ is contained in $D$ or there exists a point of $\gamma^{+}\left(y_{0}\right)$ contained in $\partial N_{1}$. The first case is impossible as before. We conclude that either there exists a point of $\gamma^{-}\left(y_{1}\right)$ contained in $\partial N_{1}$ or there exists a point of $\gamma^{+}\left(y_{0}\right)$ contained in $\partial N_{1}$. If we repeat this construction for a sequence of isolating neighborhoods $N_{i}$ shrinking to $K$ then we get that there exists a point of $K$ in the $\omega$-limit of $y_{0}$ or a point of $K$ in the $\omega^{*}$-limit of $y_{1}$. Then, as a consequence of Proposition $\left[33\right.$, there would exist a fixed point in $\mathbb{R}^{2}-K$, which cannot be 

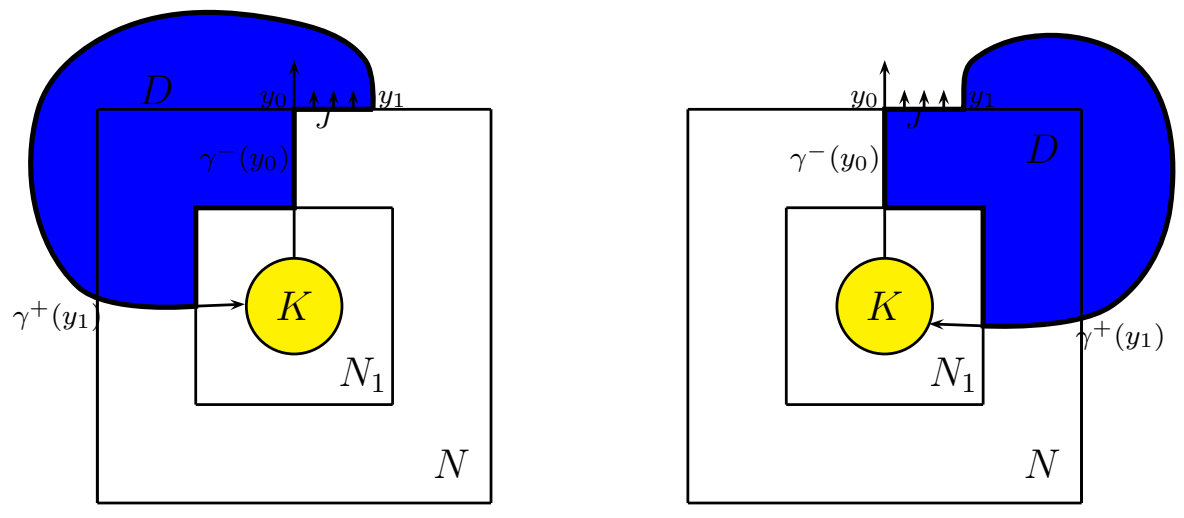

Figure 4. The region $D$

in the region of influence of $K$. It follows from this contradiction that $K$ is non-saddle and, as a consequence, a global atractor or a global repeller.

A direct consequence of Theorem 35 is the following result from [25].

Corollary 36 (Morón, Sánchez-Gabites and Sanjurjo [25]). Let $K$ be an isolated invariant continuum of a flow on $\mathbb{R}^{2}$ and suppose that $\mathcal{A}(K)=\mathbb{R}^{2}$. Then $K$ is stable and, thus, a global attractor.

\section{Robustness OF NON-SADDLE SETS}

It was shown in [14 that the property of being non-saddle is not robust, i.e. it is not preserved by continuation of isolated invariant sets. However, it turns out that there exist some relations between the preservation of certain topological properties by continuation and the preservation of the dynamical property of non-saddleness. As a matter of fact, in some situations both properties are equivalent, as we see in the following result. We introduce first the necessary definitions.

Definition 37. Suppose $\varphi_{\lambda}: M \times \mathbb{R} \rightarrow M$ is a parametrized family of flows (parametrized by $\lambda \in I$, the unit interval) in a locally compact ANR, $M$, and suppose that $K_{0}$ is an isolated non-saddle set for $\varphi_{0}$. We say that $K_{0}$ is dynamically robust if for every isolating neighborhood $N$ of $K_{0}$ there exists $\delta>0$ such that, for every $\lambda \in[0, \delta)$, the isolated invariant subset $K_{\lambda}$ of $N$ (with respect to the flow $\varphi_{\lambda}$ ) which has $N$ as an isolating neighborhood is a (non-empty) non-saddle set.

By [32, Lemma 6.2], we have that $K_{0}$ is dinamically robust if and only if there exist an isolating neighborhood $N$ of $K_{0}$ and a $\delta>0$ such that, for every $\lambda \in[0, \delta)$, the isolated invariant subset $K_{\lambda}$ of $N$ (with respect to the flow $\varphi_{\lambda}$ ) which has $N$ as an isolating neighborhood is a (non-empty) non-saddle set.

Definition 38. Suppose $\varphi_{\lambda}: M \times \mathbb{R} \rightarrow M$ is a parametrized family of flows (parametrized by $\lambda \in I$, the unit interval) in a locally compact ANR, $M$, and suppose that $K_{0}$ is an isolated invariant set for $\varphi_{0}$. We say that $K_{0}$ is topologically robust if for every isolating neighborhood $N$ of $K_{0}$ there exists $\delta>0$ such that, for every $\lambda \in[0, \delta)$, the isolated invariant subset $K_{\lambda}$ 
of $N$ (with respect to the flow $\varphi_{\lambda}$ ) which has $N$ as an isolating neighborhood has the same shape as $K_{0}$.

By [32, Lemma 6.2], we have that $K_{0}$ is topologically robust if and only if there exist an isolating neighborhood $N$ of $K_{0}$ and a $\delta>0$ such that, for every $\lambda \in[0, \delta)$, the isolated invariant subset $K_{\lambda}$ of $N$ (with respect to the flow $\varphi_{\lambda}$ ) which has $N$ as an isolating neighborhood has the same shape as $K_{0}$.

Note that when a non-saddle set is dynamically robust, this fact implies the existence of a (local) continuation made of non-saddle sets. On the other hand, if an isolated invariant set is topologically robust, then it has a (local) continuation whose members have the same shape.

Theorem 39. Let $\varphi_{\lambda}$, with $\lambda \in[0,1]$, be a differentiable parametrized family of flows on a closed and connected orientable differentiable manifold $M$ with $H^{1}(M)=\{0\}$ and $K_{0}$ be a connected isolated non-saddle set. Then $K_{0}$ is dinamically robust if and only if it is topologically robust.

Proof. It has been proved in [14] that dynamical robustness implies topological robustness. We will prove here the converse implication. Suppose that $K_{0}$ is topologically robust, i.e that $S h\left(K_{\lambda}\right)=S h\left(K_{0}\right)$ for $\lambda<\delta$. We see that since $H^{1}(M)=\{0\}$ then all $M-K_{\lambda}$ have the same number of components. Consider the terminal part of the cohomology long exact sequence

$$
\ldots \leftarrow H^{1}(M)=\{0\} \leftarrow H^{1}\left(M, M-K_{\lambda}\right) \leftarrow \widetilde{H}^{0}\left(M-K_{\lambda}\right) \leftarrow \widetilde{H}^{0}(M)=\{0\} .
$$

Then $H^{1}\left(M, M-K_{\lambda}\right) \cong \widetilde{H}^{0}\left(M-K_{\lambda}\right)$ and $H^{1}\left(M, M-K_{\lambda}\right)$ is free and finitely generated and, by the Universal Coefficient Theorem, $\operatorname{rk} H^{1}\left(M, M-K_{\lambda}\right)=\operatorname{rk} H_{1}\left(M, M-K_{\lambda}\right)$. Moreover, by Alexander duality, $H_{1}\left(M, M-K_{\lambda}\right) \cong \check{H}^{n-1}\left(K_{\lambda}\right)$ and, since Čech cohomology is a shape invariant, we have that all $\widetilde{H}^{0}\left(M-K_{\lambda}\right)$ are the same for $\lambda<\delta$ and, hence, all $M-K_{\lambda}$ have the same number of components.

Consider a connected isolating block $N$ of $K_{0}$ such that $N$ is a differentiable manifold. It was proved in [14] that $N$ is also an isolating block for all $K_{\lambda}$ with $\lambda$ sufficiently close to 0 (we may assume that this happens for all $\lambda<\delta$ ). It was also proved that the entrance and exit sets for $\varphi_{\lambda}$ are strict (i.e. without tangencies) and that they agree with those for $\varphi_{0}$. Moreover, in Theorem 25 it was proved that different components of $\partial N$ lie in different components of $M-K_{0}$. Suppose now, to get a contradiction, that $K_{\lambda}$ is saddle for some $\lambda<\delta$. Then there are sequences $x_{n} \rightarrow x \in K_{\lambda}, s_{n}<0<t_{n}$ such that $x_{n} t_{n} \in N^{0}$ and $x_{n} s_{n} \in N^{i}$. This implies that the component of $M-K_{\lambda}$ containing $x_{n}$ contains points of $N^{0}$ and $N^{i}$ and, hence, that there are two components, or more, of $\partial N$ in the component of $M-K_{\lambda}$ which contains $x_{n}$. We deduce from this that the number of components of $M-K_{\lambda}$ is less than the number of components of $M-K_{0}$. We get from this contradiction that all $K_{\lambda}$ are non-saddle for $\lambda<\delta$.

Remark 40. It is straightforward to see that Theorem 39 also holds in the case of differentiable parametrized families of flows on $\mathbb{R}^{n}, n \geq 2$.

\section{REFERENCES}

[1] K. Athanassopoulos, Explosions near isolated unstable attractors, Pacific J. Math. 210 (2003), no. 2, 201-214. 
[2] H. Barge, J.M.R. Sanjurjo, Unstable manifold, Conley index and fixed points of flows, J. Math. Anal. Appl. 420 (2014), no. 1, 835-851.

[3] A. Beck, On invariant sets, Ann. of Math. (2) 67 (1958) 99-103.

[4] N.P. Bhatia, Attraction and nonsaddle sets in dynamical systems, J. Diff. Equations 8 (1970) 229-249.

[5] N.P. Bhatia, G.P. Szego, Stability Theory of Dynamical Systems, Grundlehren der Mat. Wiss. 16, Springer, Berlin, 1970.

[6] K. Borsuk, On several problems of the theory of shape, Studies in topology: proceedings of a Conference held at Charlotte, North Carolina, march 14-16, 1974 (M. Stavrakas and K. Allen, eds.), Academic Press, 1975, pp. 67-79.

[7] K. Borsuk, Theory of Shape. Monografie Matematyczne 59, Polish Scientific Publishers, Warsaw, 1975.

[8] C. Conley, Isolated Invariant Sets and the Morse Index, CBMS Regional Conference Series in Mathematics 38 (Providence, RI: American Mathematical Society) 1978.

[9] C. Conley, R.W. Easton, Isolated invariant sets and isolating blocks, Trans. Amer. Math. Soc. 158 (1971) $35-61$.

[10] C. Conley, E. Zehnder, Morse-type index theory for flows and periodic solutions for Hamiltonian equations, Comm. Pure Appl. Math. 37 (1984) 207-253..

[11] J. Dydak, J. Segal, Shape theory. An introduction, Lecture Notes in Mathematics, 688, Springer, 1978.

[12] R.W. Easton, Isolating blocks and symbolic dynamics, J. Diff. Equations, 17 (1975) 96-118.

[13] A. Giraldo, M.A. Morón, F.R. Ruiz del Portal, J.M.R. Sanjurjo, Some duality properties of non-saddle sets, Topology Appl. 113 (2001) 51-59.

[14] A. Giraldo, J.M.R. Sanjurjo, Topological robustness of non-saddle sets, Topology Appl. 156 (2009) 19291936.

[15] C. Gutiérrez, Smoothing continuous flows on two-manifolds and recurrences, Ergod. Th. \& Dynam. Sys. 6 (1986) 17-44.

[16] M.W. Hirsch, S. Smale, R.L. Devaney, Differential Equations, Dynamical Systems, and an Introduction to Chaos. Third Edition. Elsevier/Academic Press, Amsterdam, 2013.

[17] S. Hu, Theory of retracts, Wayne State University Press, 1965.

[18] M. Izydorek, M. Styborski, Morse inequalities via Conley index theory, Topol. Methods Nonlinear Anal., 37-60, Lect. Notes Nonlinear Anal., 12, Juliusz Schauder Cent. Nonlinear Stud., Toruń, 2011.

[19] L. Kapitanski, I. Rodnianski, Shape and Morse theory of attractors. Comm. Pure Appl. Math. 53 (2000) $218-242$.

[20] J.A. Kennedy, J.A. Yorke, Bizarre topology is natural in dynamical systems, Bull. Amer. Math. Soc., 32 (1995), pp. 309-316.

[21] D. Li, A. Qi, Morse equation of attractors for nonsmooth dynamical systems, J. Diff. Equations 253 (2012) 3081-3100

[22] D.R. McMillan, Cutting off homotopies on acyclic sets, Geometric Topology: proceedings of the geometric topology Conference held at Park City, Utah, february 19-22, 1974 (L. C. Glasser and T. B. Rushing, eds.), Lecture Notes in Math., vol. 438, Springer-Verlag, 1975, pp. 343-352.

[23] D.R. Mcmillan, One-dimensional shape properties and three-manifolds, Studies in topology: proceedings of a Conferende held at Charlotte, North Carolina, march 14-16, 1974 (M. Stavrakas and K. Allen, eds.), Academic Press, 1975, pp. 367-381.

[24] P. Mendelson, On unstable attractors, Bol. Soc. Mat. Mexicana (2) 5 (1960), 270-276.

[25] M.A. Morón, J.J. Sánchez Gabites and J.M.R. Sanjurjo, Topology and dynamics of unstable attractors, Fund. Math. 197 (2007) 239-252.

[26] J. Palis, W. de Melo, Geometric Theory of Dynamical Systems. An Introduction. Springer-Verlag, New York-Berlin 1982.

[27] S.Y. Pilyugin, Introduction to Structurably Stable Systems of Differential Equations, Birkhaüser Verlag, 1992.

[28] I. Richards, On the classification of noncompact surfaces, Trans. Amer. Math. Soc. 106 (1963) 259-269.

[29] J.W. Robbin, D. Salamon, Dynamical systems, shape theory and the Conley index, in: Charles Conley Memorial Volume, Ergodic Theory Dynam. Systems 8* (1988) 375-393. 
[30] J.C. Robinson, Infinite-Dimensional Dynamical Systems. An Introduction to Dissipative Parabolic PDEs and the Theory of Global Attractors, Cambridge Texts in Applied Mathematics. Cambridge University Press, Cambridge, 2001.

[31] J.C. Robinson, Global attractors: topology and finite-dimensional dynamics, J. Dynam. Differential Equations 11 (1999), no. 3, 557-581.

[32] D. Salamon, Connected simple systems and the Conley index of isolated invariant sets, Trans. Amer. Math. Soc. 291 (1985) 1-41.

[33] J.J. Sánchez-Gabites, Unstable attractors in manifolds, Trans. Amer. Math. Soc. 362 (2010) 3563-3589.

[34] J.M.R. Sanjurjo, An intrinsic description of shape, Trans. Amer. Math. Soc. 329 (1992) 625-636.

[35] J.M.R. Sanjurjo, Multihomotopy, Cech spaces of loops and shape groups, Proc. London Math. Soc. 69 (1994) 330-344.

[36] J.M.R. Sanjurjo, On the structure of uniform attractors, J. Math. Anal. Appl. 192 (1995) 519-528.

[37] J.M.R. Sanjurjo, Global topological properties of the Hopf bifurcation, J. Diff. Equations, 243 (2007) $238-255$.

[38] J.M.R. Sanjurjo, Stability, attraction and shape: a topological study of flows, Topol. Methods Nonlinear Anal., 93-122, Lect. Notes Nonlinear Anal., 12, Juliusz Schauder Cent. Nonlinear Stud., Toruń, 2011.

[39] E.H. Spanier, Algebraic topology, McGraw-Hill Book Co., 1966.

[40] R. Thom, Quelques propiétés globales des variétés différentiables, Comment. Math. Helv. 28 (1954) $17-86$.

[41] T. Ura, On the flow outside a closed invariant set; stability, relative stability and saddle sets, Contrib. Differential Equations 3 (1963), 249-294.

[42] T. Ura, I. Kimura, Sur le courant exterieur a une region invariante. Theoreme de Bendixson, Comm. Mat. Univ. Sancii Pauli 8 (1960) 23-39.

[43] J. Wang, D. Li, J. Duan, On the shape Conley index theory of semiflows on complete metric spaces, Disc. Cont. Dyn. Sys. 36 (2016) 1629-1647.

E.T.S. Ingenieros informáticos, Universidad Politécnica de Madrid, Madrid 28660, Spain

E-mail address: h.barge@upm.es

Facultad de C. C. Matemáticas, Universidad Complutense de Madrid, Madrid 28040, Spain

E-mail address: email:jose_sanjurjo@mat.ucm.es 